This item was submitted to Loughborough's Research Repository by the author.

Items in Figshare are protected by copyright, with all rights reserved, unless otherwise indicated.

\title{
Communication-aware trajectory planning for unmanned aerial vehicles in urban environments
}

\section{PLEASE CITE THE PUBLISHED VERSION}

https://doi.org/10.2514/1.G003099

\section{PUBLISHER}

(c) American Institute of Aeronautics and Astronautics

\section{VERSION}

AM (Accepted Manuscript)

\section{PUBLISHER STATEMENT}

This work is made available according to the conditions of the Creative Commons Attribution-NonCommercialNoDerivatives 4.0 International (CC BY-NC-ND 4.0) licence. Full details of this licence are available at: https://creativecommons.org/licenses/by-nc-nd/4.0/

\section{LICENCE}

CC BY-NC-ND 4.0

\section{REPOSITORY RECORD}

Oh, Hyondong, Hyo-Sang Shin, Seungkeun Kim, and Wen-Hua Chen. 2018. "Communication-aware Trajectory Planning for Unmanned Aerial Vehicles in Urban Environments". figshare. https://hdl.handle.net/2134/33229. 


\title{
Communication-Aware Trajectory Planning for Unmanned Aerial Vehicles in Urban Environments
}

\author{
Hyondong $\mathrm{Oh}^{*}$, \\ Ulsan National Institute of Science and Technology, Ulsan, 44919, Republic of Korea \\ Hyo-Sang Shin $^{+}$, \\ Cranfield University, Cranfield, Bedfordshire, MK43 OAL, United Kingdom \\ Seungkeun $\mathrm{Kim}^{\sharp}$, \\ Chungnam National University, 99 Daehak-ro, Daejeon 305-764, Republic of Korea \\ and Wen-Hua Chen** \\ Loughborough University, Loughborough, Leicestershire, LE11 3TU, United Kingdom
}

\section{INTRODUCTION}

Maintaining communication among mobile agents in a networked team is challenging due to limited bandwidth, maximum communication range, transmission power, and physical obscuration or occlusion in the mission environment. With the advent of lightweight, robust and autonomous platforms as well as wireless networking technologies, it becomes feasible to use small unmanned aerial vehicles (UAVs) as communication relay nodes under limited satellite communication environments [1]. This communication relay UAV could allow a ground operator/system to have sufficient datalink to effectively see beyond the communication range and over the horizon/buildings where traditional methods fail. The relay UAV can also be used to transmit/share critical information efficiently from an operator to an end-user or between vehicles.

\footnotetext{
*Assistant Professor, School of Mechanical, Aerospace and Nuclear Engineering, AIAA Member; h.oh@unist.ac.kr.

${ }^{+}$Reader, Institute of Aerospace Sciences, AIAA Member; h.shin@cranfield.ac.uk.

${ }^{\sharp}$ Associate Professor, Department of Aerospace Engineering, AIAA Senior Member; skim78@cnu.ac.kr.

** Professor, Department of Aeronautical and Automotive Engineering; w.chen@lboro.ac.u.
} 
To maintain coverage of the large operation area, relay UAVs should have the ability to fly position themselves in a way to ensure the successful and efficient transmission of critical data in dynamic and challenging mission environments. For this effective trajectory planning of the relay UAV considering communication constraints, it is essential to predict the communication performance in the problem domain in order to assess the feasibility of the trajectories. To make these predictions, a certain model or measured metric of the communication environment is required, which can be largely categorised into [2]: i) model-based approaches where a model of the communication environment is used to predict the communication performance of the networked team and ii) measurement-based approaches which make use of online communication quality measurements.

Model-based approaches can be further categorised into: i) range-only, ii) range and visibility, iii) channel propagation. Many traditional model-based works considered range-only communication constraints [3]-[6], while a few of them considered visibility (i.e. if there is any obstruction between agents which is often termed as line-of-sight (LOS)) as well as range [7]. For more realistic communication modelling, a channel propagation model is used, which is based on a deterministic part of an exponential distance path loss model and on a stochastic part describing small-scale fading by a Rayleigh distribution; this model provides the probability of successful communication in the network [8]-[12]. Some of recent channel propagation models also considered LOS as the availability of the LOS condition could affect communication quality significantly [13]-[16]. Note that, for model-based approaches, positional information of all agents is required.

For measurement-based approaches, the signal-to-noise ratio (SNR) or similar channel metrics are assumed to be measured, and a gradient following method [17]-[19] is used to guide the vehicle towards the area which gradually produces higher SNR, without much relying on prespecified communication model and location information. There are recent works on a hybrid approach combining the model- and measurement-based methods, which corrects a modelling error with measured communication quality and a learning approach [20]-[22].

Although there are existing works on hardware development for airborne radio communication or low-cost/power communication protocol such as Zigbee and Wifi, limited research works on high-level autonomous mission or path/trajectory planning are reported to be used for UAV communication relay. It is also worthwhile to note that most existing aforementioned 
approaches using UAVs as communication relay employ a single multi-rotor UAV (e.g. helicopter or quadrotor which can hover) and a simple distance-based communication model. These might be vulnerable to the failure of the relay UAV or inefficient for uncertain and dynamic environments. If ground mobile nodes move dynamically, then an optimal networking structure and the corresponding desired relay UAV position to efficiently share/transmit data between them might change significantly, which might be difficult to be followed by the multi-rotor UAV due to its limited mobility. Besides, many of them consider only a 2-D (two dimensional) and open field environment where the UAV flies at a fixed altitude and there are no buildings or obstacles.

To address above issues, this paper proposes a 3-D communication-aware trajectory planning approach to guide small multiple fixed-wing UAVs continuously to relay information (e.g. live surveillance feed) between the ground control station (GCS) and friendly ground vehicles (termed as a convoy hereafter) moving in an urban area. In particular, we focus on maintaining a direct LOS and the minimum distance between relay UAVs and the ground convoy. The availability of the geometrical LOS does not necessarily mean that the RF LOS condition is satisfied. There may be a case where direct path penetrates some light obstacles (termed as the obstructed LOS case [15]), or multi-path effects make it not so straightforward to assume that maintaining LOS is a good measure of communication performance. However, as the LOS condition is one of the most primary factors affecting the communication signal strength as identified in [13]-[16], this study focuses on the LOS condition in order to ensure the required communication quality.

In complex urban environments, maintaining the LOS is quite challenging as there are a large number of differently-shaped buildings and obstacles which might obstruct the LOS [23]. To this end, the 3-D visibility polytope, termed as a communication-feasible area is first computed, which has a direct LOS from the moving convoy to relay UAVs within the maximum communication range. Relay UAVs are then controlled to stay in this area while being as close as possible to the convoy for better communication quality. Considering that the communication-feasible area is dynamically changing according to the movement of a convoy and there are kinematic constraints (speed and turning rates) on fixed-wing UAVs, a nonlinear model predictive control (NMPC)-based trajectory planning algorithm is proposed by extending our previous work [24] into 3-D. Kinematic constraints of the UAV and collision avoidance requirements between UAVs or between the UAV and obstacles (e.g. no-fly zone) can be easily incorporated into the NMPC framework. Besides, since the NMPC optimises UAV control commands periodically by 
utilising a 3-D UAV dynamic model, the future convoy positions and the corresponding future communication-feasible areas, it can incorporate the change in the predicted communicationfeasible area over the horizon [25]. Therefore, it is expected that the proposed NMPC-based algorithm provides a good performance in terms of making UAVs stay in the communicationfeasible area in urban environments. Numerical simulation results confirm the benefit of using the proposed approach in a complex urban environment, compared with the broadly-used Lyapunov vector field guidance [26], [27].

The structure of this paper is given as: Section II presents the assumptions and overview of the proposed algorithm. Section III describes communication feasible area and desired loitering orbit generation, followed by the convoy following trajectory planning algorithm based on the NMPC in Section IV. Section V presents numerical simulation results for sample convoy following scenarios. Lastly, conclusions are presented in Section VI.

\section{Communication-Aware Trajectory Planning Approach Overview}

There are several assumptions made in the study. Firstly, the urban map environment is assumed to be known. Vehicles in a convoy are assumed to be able to communicate with each other, so they are regarded as one convoy unit. Trajectory of the convoy is known (or can be estimated from the current time to a few time steps further ahead) to the GCS. A convoy is assumed to move on a 2-D plane (i.e. altitude is zero). Relay UAVs share a known global coordinate system for their own and convoy position. Communication between nodes (relay UAVs, a convoy and a GCS) is limited by the communication range and visibility, so if they are within a certain distance and there exists a direct LOS (not obstructed by buildings), it is assumed that they can communicate with each other.

Figure 1 shows an illustrative example of a communication-aware convoy following scenario using multiple UAVs in an urban environment. Convoy following UAVs need to fly within the communication-feasible area (green shaded region which satisfies the communication requirements between the UAV and the ground convoy) in order to relay information from the ground moving convoy to the GCS. Note that one or more intermediate relay UAVs might be required to connect the GCS and convoy following UAVs in case they are beyond the normal communication range or a direct LOS between them is obstructed. Note that the focus of this work is in the trajectory designing of convoy following UAVs, so the detailed deployment plan for intermediate 
relay UAVs is not considered in depth.

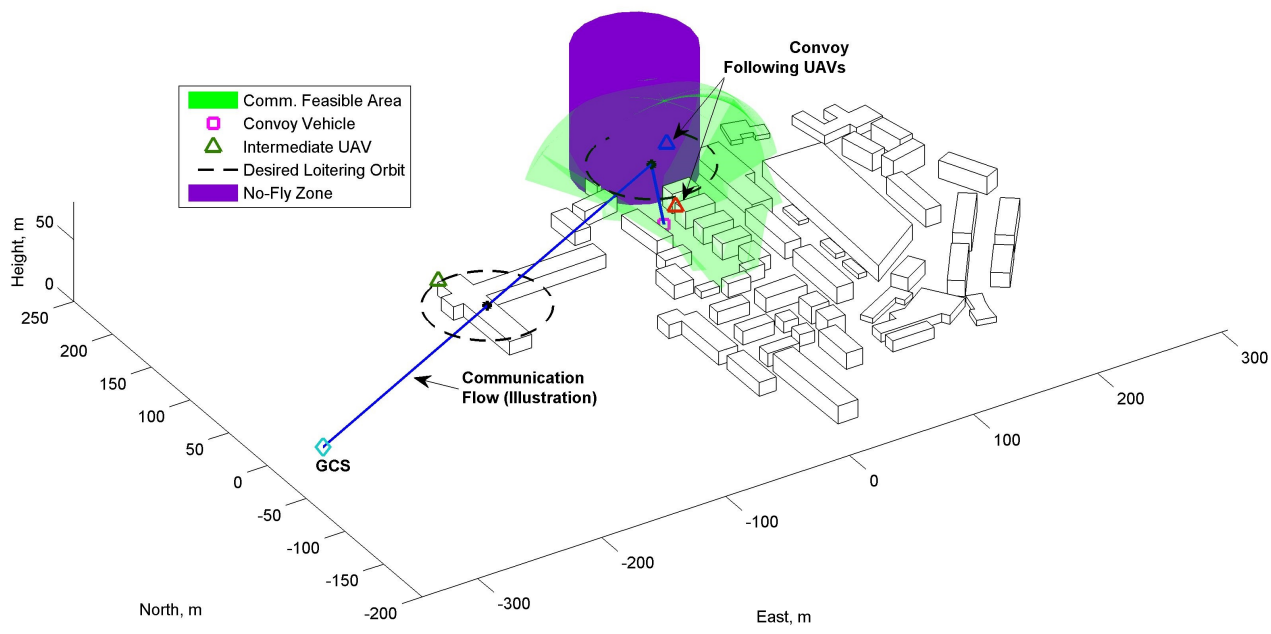

Fig. 1. An illustrative scenario of a communication-aware convoy following mission in an urban environment.

Figure 2 shows the flow chart of the proposed approach on communication-aware convoy following trajectory planning. Firstly, a 3-D communication-feasible area is obtained for the current convoy location in the region of interest. Then, to make the fixed-wing UAV fly within this area while being as close as possible to the ground convoy, an appropriate desired loitering orbit is determined. Lastly, a nonlinear model predictive control (NMPC)-based trajectory planning algorithm is designed to make the UAV follow the desired loitering orbit by utilising the future convoy positions and the corresponding communication-feasible area while considering UAVs' speed and turning rate constraints.

\section{Communication-Feasible Area And Orbit Generation}

A. 3-D Visibility region with communication range

An urban environment space can be mathematically defined as:

$$
\mathbf{X}_{E}=\left\{(x, y, z) \in \mathbb{R}^{3} \mid z \geq 0\right\}
$$

with buildings in it, represented by a polyhedron's body $\mathbf{B}^{o}$

$$
\mathbf{B}=\bigcup_{o=1}^{N_{o}} \mathbf{B}^{o}
$$




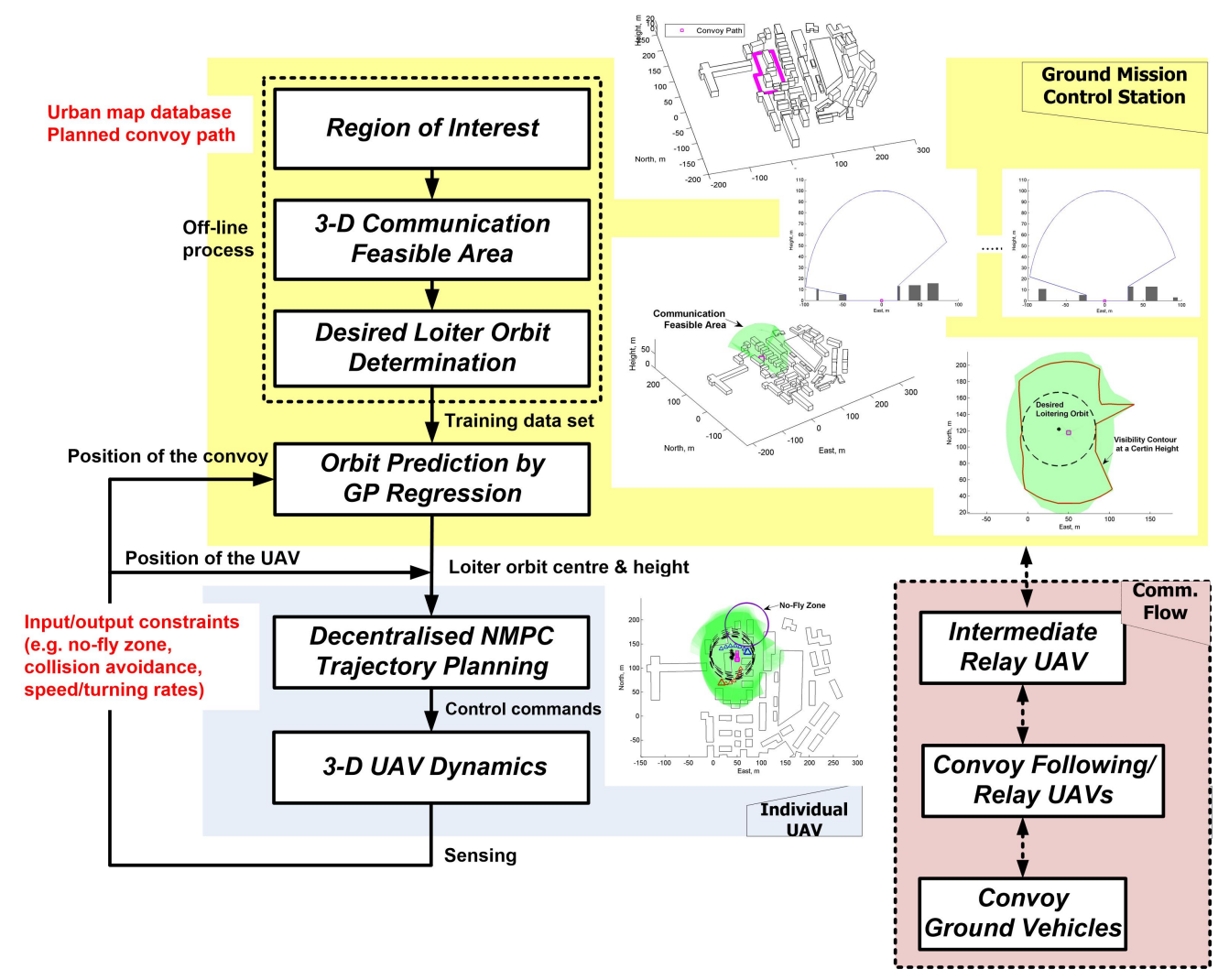

Fig. 2. Flow chart of the proposed communication-aware convoy following trajectory planning process.

where $N_{o}$ is the number of buildings. Then, a 3-D communication-feasible area $\mathbf{X}_{C F} \subset \mathbf{X}_{E}$ for the possible position of the ground convoy $\mathbf{x}_{c v}=\left(x_{c v}, y_{c v}, z_{c v}\right) \in \mathbb{R}^{3}$ considering the line-ofsight (LOS) condition to the convoy and the limited communication range $r_{c o m}^{\max }$ can be defined as:

$$
\mathbf{X}_{C F}=\left\{(x, y, z) \in \mathbf{X}_{E}\|\|(x, y, z)-\mathbf{x}_{c v} \|_{2} \leq r_{c o m}^{\max }, \mathbf{x}_{c v}+k(x, y, z) \notin B, \forall k \geq 0\right\}
$$

This represents all the points on straight lines from the convoy location $\mathbf{x}_{c v}$ to the arbitrary point in the environment within the limited communication range and not obstructed by buildings. Note that the communication-feasible area $\mathbf{X}_{C F}$ in Eq. (3) excludes a region where LOS communication is in fact possible with the convoy as the UAV is close to it, even though, by continuing the LOS ray beyond the UAV, it might intersect with a building. However, as we assume that the UAV flies above the buildings not between them, this exclusion does not affect our communication-aware trajectory planning process. 
Obtaining $\mathbf{X}_{C F}$ exactly at an arbitrary convoy position $\mathbf{x}_{c v}$ where there are a large number of buildings in the environment would be computationally intractable. Thus, in practice, $\tilde{\mathbf{X}}_{c v}$ needs to be approximately computed by combining several discrete 2-D visibility polygons as used in [28]. For a given convoy position, the 2-D cross section of visibility polygons with a limited communication range at a certain azimuth angle (e.g. East-Height cross section as shown in Fig. 3(a)) can be obtained while considering LOS block by corresponding buildings. For this, VisiLibity [29], a free open source C++ library for 2-D floating-point visibility algorithms, is used among other library functions. Then, joining and patching several vertical cross sections over 180 degrees sampled at a fixed angle interval results in the 3-D communication-feasible area (visibility polytope) as shown in Fig. 3(b). Note that the sampling angle interval needs to be carefully determined considering the system requirement as it is a trade-off between the computation time, the data storage and the resolution of the communication-feasible area. For instance, if the sampling interval is too large, e.g. 30 degrees, then only six cross sections would be needed to build the entire 3-D area; however, this area representation might not be dense enough to be able to detect LOS block by buildings whose dimension is smaller than the one from the sampling angle.

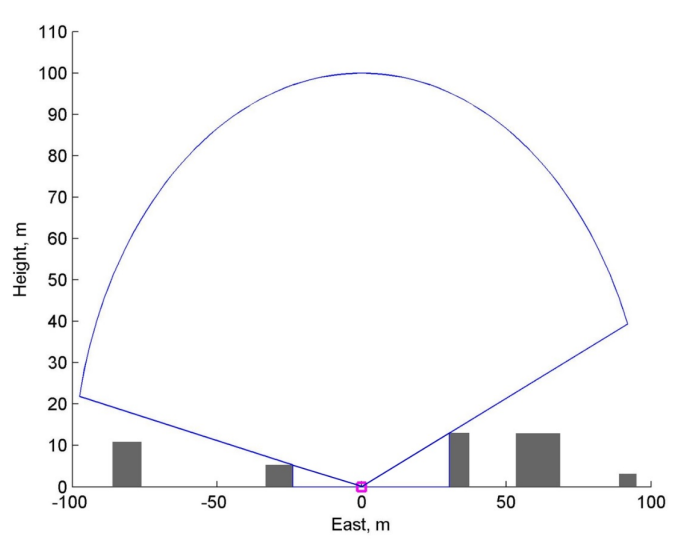

(a) 2-D

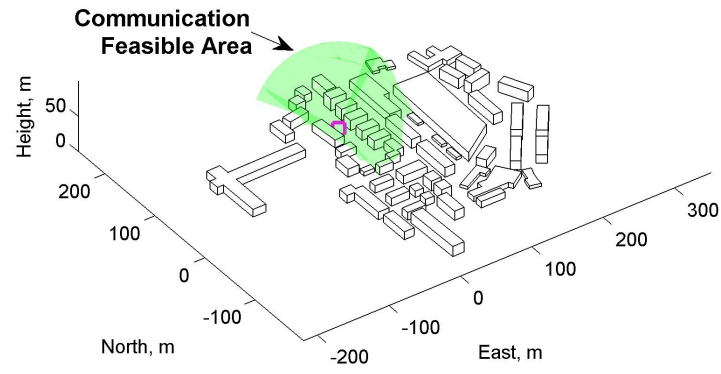

(b) 3-D

Fig. 3. Communication-feasible area generation.

The benefits of calculating the communication-feasible area for given convoy positions compared with examining if certain candidate UAV positions in the air are within the communication range while having an occlusion-free LOS to the ground convoy are manifolds: i) the proposed 
approach requires fewer calculations as this generates the communication-feasible area from 2-D convoy positions rather than checking the communication requirements (range and LOS) for all 3-D UAV positions in the air; ii) the communication-feasible area can be pre-computed and stored for the convoy path or even for entire map grid positions, as will be explained in Section III.C; and iii) this area needs to be computed once for the target location regardless of the number of UAVs involved. Besides, the communication-feasible area provides the UAV with more flexibility as the UAV has the information on how much space is available for manoeuvring. Our work builds upon these benefits of communication-feasible area generation.

\section{B. Desired loitering orbit determination}

Once the communication-feasible area is generated, then UAVs should fly within the generated area in order to maintain the communication (and the LOS) to the convoy at all times. Although there might be several ways to do so, we adopt a loitering (or standoff) orbit tracking concept [24], [30]-[32] to this problem, which makes the UAV follow a loitering orbit determined by the communication-feasible area. This has several benefits over other approaches (e.g. just flying within the area [28] or following the exact area boundary): it prevents sudden change of the flight course, so it is efficient under dynamic constraints of a fixed-wing UAV; path or behaviour is predictable to a certain extent as it loiters around a known point; straightforward collision avoidance between UAVs if multiple UAVs are involved by enforcing angular separation on the same orbit; orbiting around the ground convoy would allow to gather more information; and being able to be as close as possible to the convoy all the time (hence, better communication quality and network connectivity) while satisfying the movement constraints.

Note that, if the UAV flies at a low altitude, then it might not be able to stay within the corresponding communication-feasible area due to the turning rate (or minimum turning radius) limitation. For instance, at $h=h_{1}$ in Fig. 4, the area is too small to contain a certain size of the desired loitering orbit (represented as the dashed black circle). On the other hand, at a high altitude $h=h_{2}$, even though there would be enough space for the UAV to move around but the distance between the ground convoy and the UAV becomes large. Consequently, finding the minimum height $h_{d}$ and a loitering centre $\bar{r}_{c t, d}$ in the generated communication-feasible area in 
which the UAV can stay becomes a necessary task. This is formulated as:

$$
h_{d}=\min h
$$

$$
\begin{array}{ll}
\text { s.t. } & \left\|\overline{\mathbf{r}}_{c t}-\mathbf{B}_{p t, i}\left(\mathbf{X}_{C F}^{h}\right)\right\|_{2} \geq r_{\text {turn }}^{\text {min }}+\varepsilon_{1}=r_{d} \forall i \in\left\{1, \ldots, N_{p}\right\}, \\
& h \geq h_{\text {build }}^{\text {max }}+\varepsilon_{2},
\end{array}
$$

where $\|\cdot\|_{2}$ represents a L2-norm, $\mathbf{B}_{p t, i}\left(\mathbf{X}_{C F}^{h}\right) \in\left\{\mathbf{B}_{p t, 1}, \cdots, \mathbf{B}_{p t, N_{p}}\right\} \subset \mathbb{R}^{2 \times N_{p}}$ represents the set of discretised boundary points of the communication-feasible area at a height of $h$ (i.e. $\left.\mathbf{X}_{C F}^{h}=\left\{(x, y, z) \in \mathbf{X}_{C F} \mid z=h\right\}\right), N_{p}$ is the number of the boundary points and $\overline{\mathbf{r}}_{c t}=$ $\sum_{i=1}^{N_{p}} \mathbf{B}_{p t, i}\left(\mathbf{X}_{C F}^{h}\right) / N_{p}$ is the mean position of them. $r_{\text {turn }}^{\text {min }}$ is the minimum turning radius of the $\mathrm{UAV}$ and $\varepsilon_{1}$ is a positive margin, so $r_{d}=r_{\text {turn }}^{\min }+\varepsilon$ is a desired loitering orbit radius. $h_{\text {build }}^{\text {max }}+\varepsilon_{2}$ represents the safe flight altitude which is higher than the maximum height of the buildings in the communication-feasible area. Once $h_{d}$ is found, the corresponding loitering centre $\bar{r}_{c t, d}$ can be easily computed.

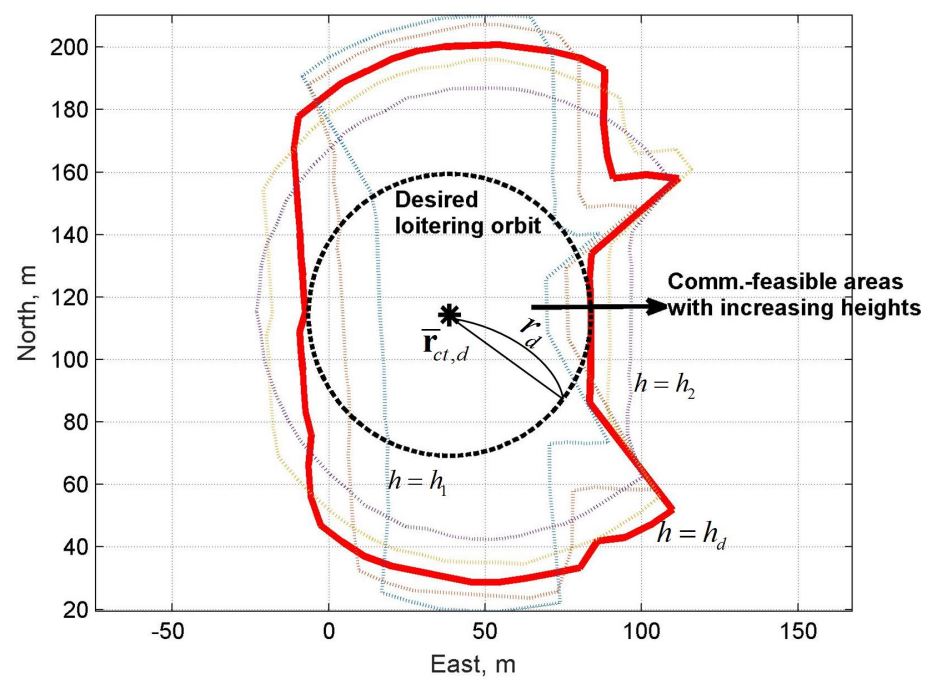

Fig. 4. The cross sections of the communication-feasible area at different heights for the same ground position.

\section{Gaussian Process regression on desired loitering orbits}

The real-time computation of the communication-feasible area and the corresponding desired loitering orbit for a convoy path might be computationally expensive due to the complicated 
building geometry of the urban area. In particular, if the online computation capacity of the ground control station is limited, this process could be partially made offline by using machine learning techniques. Using a set of computed desired loitering orbits for sampled ground convoy positions in the region of interest as training data, the machine learning approach can provide the approximated relationship between an arbitrary ground convoy position and the desired loitering orbit for that location. With this trained (learned) relationship, the desired loitering orbit to be followed by UAVs can be computed quickly for a convoy path or any arbitrary ground position in real time.

In order to investigate the feasibility of this concept, this paper utilises the Gaussian Process regression (GPR) [33] among other machine learning techniques. However, this does not restrict implementation of other machine learning algorithms. The Gaussian Process (GP) can be viewed as a Gaussian distribution over functions, and it can be used to infer or predict function values at a finite set of test points using the observed data. Regression using the GP is briefly explained as follows.

Firstly, a standard regression model is defined as $y_{G P}=f(\mathbf{x})+\epsilon$, where $\mathbf{x}$ is an input vector, and $y_{G P}$ is a scalar output. The noise $\epsilon$ is assumed to be an independent and identically distributed Gaussian distribution with zero mean and variance $\sigma^{2}$. Then, the Gaussian Process $f(\mathbf{x})$ is specified by its mean function $m(\mathbf{x})=\mathbb{E}[f(\mathbf{x})]$ and the covariance function $k\left(\mathbf{x}, \mathbf{x}^{\prime}\right)=$ $\mathbb{E}\left[(f(\mathbf{x})-m(\mathbf{x}))\left(f\left(\mathbf{x}^{\prime}\right)-m\left(\mathbf{x}^{\prime}\right)\right)\right]$. Since this study assumes zero-mean GP, the process can be expressed as $f(\mathbf{x}) \sim G P\left(0, k\left(\mathbf{x}, \mathbf{x}^{\prime}\right)\right)$. A training set with $N_{t}$ observations is expressed as $\mathcal{D}=$ $\left\{\left(\mathbf{x}_{n}, y_{G P, n}\right) \mid n=1, \cdots, N_{t}\right\}=\left\{\mathbf{X}, \mathbf{y}_{G P}\right\}$, and the following squared exponential covariance function is used:

$$
k\left(\mathbf{x}, \mathbf{x}^{\prime}\right)=\sigma_{f}^{2} \exp \left(-\frac{1}{2}\left(\mathbf{x}-\mathbf{x}^{\prime}\right)^{\top} \sum^{-1}\left(\mathbf{x}-\mathbf{x}^{\prime}\right)\right)
$$

where $\sigma_{f}$ determines the magnitude, and $\sum=l^{-2} I$ is an isotropic covariance function.

Given the GP model and the covariance function above, the fitness of this model to the training set $\mathcal{D}$ can be evaluated using the marginal likelihood conditioned on the hyper-parameters $\theta$ (i.e. the parameters of the covariance function):

$$
\begin{aligned}
& \log p\left(\mathbf{y}_{G P} \mid \mathbf{X}, \theta\right)= \\
& -\frac{1}{2} \mathbf{y}_{G P}^{\top} \mathbf{K}_{y}^{-1} \mathbf{y}_{G P}-\frac{1}{2} \log \left|\mathbf{K}_{y}\right|-\frac{N_{t}}{2} \log 2 \pi
\end{aligned}
$$


where $\mathbf{K}_{y}=\mathbf{K}+\sigma^{2} I$ and $\mathbf{K}=k(\mathbf{X}, \mathbf{X})$. The hyper-parameters are optimised to provide good predictions using the partial derivatives of Eq. (6) with respect to the hyper-parameters using a gradient-based optimiser. Note that these hyperparameters are fixed once they are optimised with the training set.

Given the training set $\mathcal{D}$, the covariance function with the trained hyperparameters, and a test input vector $\mathrm{x}_{*}$, the predictive distribution for the GPR can be computed as:

$$
f_{*} \mid \mathbf{X}, \mathbf{y}_{G P}, \mathbf{x}_{*} \sim \mathcal{N}\left(\bar{f}_{*}, \mathbb{V}\left[f_{*}\right]\right)
$$

where the mean and variance are defined as:

$$
\begin{aligned}
\bar{f}_{*} & =\mathbf{k}_{*}^{\top}\left(\mathbf{K}+\sigma_{n}^{2} I\right)^{-1} \mathbf{y}_{G P} \\
\mathbb{V}\left[f_{*}\right] & =k\left(\mathbf{x}_{*}, \mathbf{x}_{*}\right)-\mathbf{k}_{*}^{\top}\left(\mathbf{K}+\sigma_{n}^{2} I\right)^{-1} \mathbf{k}_{*}
\end{aligned}
$$

where $\mathbf{k}_{*}$ denotes the vector of covariance between the test and the training points.

In this study, the centre of the desired loitering orbit $\left(\overline{\mathbf{r}}_{c t, d}\right)$ and its height $\left(h_{d}\right)$ at certain points of the ground vehicle (or convoy) ( $4 \mathrm{~m}$ by $4 \mathrm{~m}$ grids) in the region of interest are used as a training data set. Figure 5 shows the example result of the GPR on the desired loitering orbit height using $1 \mathrm{~m}$ by $1 \mathrm{~m}$ grids over a certain area as test points, and it shows the higher height around narrow roads as expected. Note that once the GPR process (in particular, hyperparameter optimisation) is done offline, the predicted output $\bar{f}_{*}$ (i.e. the desired orbit centre and height) at the current convoy position can be obtained in real time using Eq. (8).

\section{NMPC-BASED LOITERING ORBIT TRACKING GUIDANCE}

This section presents a three-dimensional (3-D) dynamic model of the UAV and nonlinear model predictive control (NMPC)-based convoy following trajectory planning to follow the desired loitering orbit accurately. Since the desired loitering orbit is dynamically changing and there are kinematic constraints on the UAV as well as collision and obstacle avoidance requirements, the NMPC framework is used for loitering orbit following. It optimises the control command by utilising multiple time-step ahead information of the ground convoy and the corresponding communication-feasible area to provide a sub-optimal tracking performance. Note that the NMPC problem is formulated for a pair of UAVs to have redundancy in case that one UAV loses communication (i.e. going outside of the feasible area) by maintaining a desired 


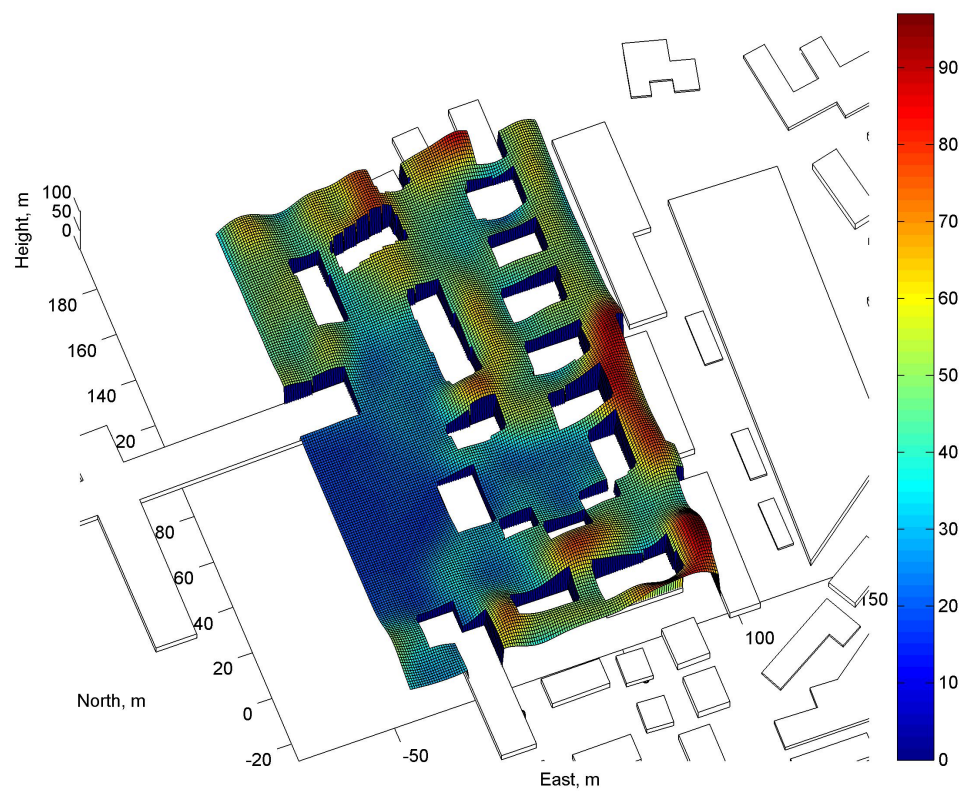

Fig. 5. The example result of the GPR on the desired height using 1 by $1 \mathrm{~m}$ grid test points over an area of interest.

fixed angular separation between them. An adpative angular seperation or different cooperation strategy remains as future work.

\section{A. 3-D UAV dynamic model}

Assuming each UAV has a low-level flight controller such as stability/controllability augmentation system for heading, flight path and velocity hold functions, this study aims to design guidance inputs to this low-level controller for loiter orbit tracking. Consider a 3-D UAV kinematic model 
by extending a 2-D model given in [24]:

$$
\left(\begin{array}{c}
\dot{x} \\
\dot{y} \\
\dot{z} \\
\dot{\chi} \\
\dot{\gamma} \\
\dot{v} \\
\dot{\omega}_{\chi} \\
\dot{\omega}_{\gamma}
\end{array}\right)=f(\mathbf{x}, \mathbf{u})=\left(\begin{array}{c}
v \cos \chi \cos \gamma \\
v \sin \chi \cos \gamma \\
v \sin \gamma \\
\omega_{\chi} \\
\omega_{\gamma} \\
-\frac{1}{\tau_{v}} v+\frac{1}{\tau_{v}} u_{v} \\
-\frac{1}{\tau_{\omega_{\chi}}} \omega_{\chi}+\frac{1}{\tau_{\omega_{\chi}}} u_{\omega_{\chi}} \\
-\frac{1}{\tau_{\omega_{\gamma}}} \omega_{\gamma}+\frac{1}{\tau_{\omega_{\gamma}}} u_{\omega_{\gamma}}
\end{array}\right)
$$

where $\mathbf{x}=\left(x, y, z, \chi, \gamma, v, \omega_{\chi}, \omega_{\gamma}\right)^{T}$ are the inertial 3-D position, heading (or course) angle, flight path angle, speed, and yaw and pitch rate of the UAV, respectively. $\tau_{v}, \tau_{\omega_{\chi}}$, and $\tau_{\omega_{\gamma}}$ are time constants for considering the dynamics of corresponding channels. $\mathbf{u}=\left(u_{v}, u_{\omega_{\chi}}, u_{\omega_{\gamma}}\right)^{T}$ are the commanded speed, turning rate and pitch rate constrained by the following dynamic limits of the fixed-wing UAV: $\left|u_{v}-v_{0}\right| \leq v_{\max },\left|u_{\omega_{\chi}}\right| \leq \omega_{\chi, \max }$, and $\left|u_{\omega_{\gamma}}\right| \leq \omega_{\gamma, \max }$ where $v_{0}$ is a nominal speed of the UAV. The continuous UAV model in (10) can be discretised by Euler integration into:

$$
\mathbf{x}_{k+1}=f_{d}\left(\mathbf{x}_{k}, \mathbf{u}_{k}\right)=\mathbf{x}_{k}+T_{s} f\left(\mathbf{x}_{k}, \mathbf{u}_{k}\right)
$$

where $\mathbf{x}_{k}=\left(x_{k}, y_{k}, z_{k}, \chi_{k}, \gamma_{k}, v_{k}, \omega_{\chi k}, \omega_{\gamma k}\right)^{T}, \mathbf{u}_{k}=\left(u_{v k}, u_{\omega_{\chi} k}, u_{\omega_{\gamma} k}\right)^{T}$, and $T_{s}$ is a sampling time.

\section{B. Definition of performance index and constraints}

The geometry between the UAV, the pair UAV, and the desired loitering orbit (with a centre point) at the desired height considered in this study is shown in Fig. 6. The basic aim of loitering orbit tracking is to maintain a distance $\left|\boldsymbol{r}_{k}\right|$ between the UAV and the centre point to $r_{d}$, a height of UAVs to a desired height $h_{d}$ and a relative phase angle $\Delta \gamma$ between UAVs to a desired value concurrently. Here, the relative phase angle is useful for collision avoidance between UAVs flying on the same desired loitering orbit while providing more persistent communication connectivity to the ground vehicle. Then, loitering orbit tracking can be formulated as a nonlinear model predictive control problem to find a control input sequence $U_{k}=\left\{\mathbf{u}_{0}, \mathbf{u}_{1}, \ldots, \mathbf{u}_{N-1}\right\}$ that 


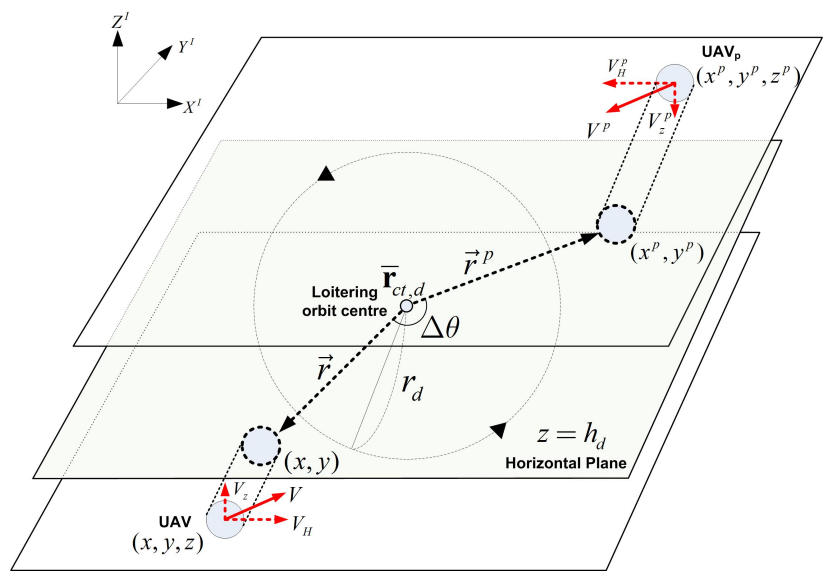

Fig. 6. The geometry between the UAV, the pair UAV, and the desired loitering orbit at the height of $h_{d}$.

minimises the following performance index:

$$
\begin{aligned}
J= & \phi\left(\tilde{r}_{N}, \tilde{d}_{N}, \tilde{h}_{N}\right)+\sum_{k=0}^{N-1} L\left(\tilde{r}_{k}, \tilde{d}_{k}, \tilde{h}_{k}, \mathbf{u}_{\mathbf{k}}\right) \\
\phi\left(\tilde{r}_{N}, \tilde{d}_{N}, \tilde{h}_{N}\right)= & \frac{1}{2}\left(p_{r} \tilde{r}_{N}^{2}+p_{d} \tilde{d}_{N}^{2}+p_{h} \tilde{h}_{N}^{2}\right) \\
L\left(\tilde{r}_{k}, \tilde{d}_{k}, \mathbf{u}_{\mathbf{k}}\right)= & \frac{1}{2}\left\{q_{r} \tilde{r}_{k}+q_{d} \tilde{d}_{k}^{2}+q_{h} \tilde{h}_{k}^{2}+\right. \\
& \left.r_{v}\left(\frac{u_{v k}-v_{0}}{v_{\max }}\right)^{2}+r_{\omega_{\chi}}\left(\frac{u_{\omega_{\chi} k}-\frac{v_{0}}{r_{d}}}{\omega_{\chi, \max }}\right)^{2}+r_{\omega_{\gamma}}\left(\frac{u_{\omega_{\gamma} k}}{\omega_{\gamma, \max }}\right)^{2}\right\}
\end{aligned}
$$

where

$$
\begin{aligned}
\tilde{r}_{k} & =\frac{r_{d}^{2}-\left|\boldsymbol{r}_{k}\right|^{2}}{r_{d}^{2}} \\
\tilde{d}_{k} & =\frac{\boldsymbol{r}_{k}^{T} \boldsymbol{r}_{k}^{p}+\left|\boldsymbol{r}_{k}\right|\left|\boldsymbol{r}_{k}^{p}\right|}{r_{d}^{2}} \\
\tilde{h}_{k} & =\frac{h_{d}^{2}-z_{k}^{2}}{h_{d}^{2}}
\end{aligned}
$$

Here, $\boldsymbol{r}_{k}$ and $\boldsymbol{r}_{k}^{p}$ represent the relative vectors from the loitering orbit centre to the positions of the current UAV and its pair UAV, respectively. Also, $r_{d}$ is a desired loitering distance from the UAVs to the loitering orbit centre, $h_{d}$ is a desired height for UAVs to maintain, $N$ is the length of receding horizon, $v_{0}$ is a nominal speed of UAVs, and $\frac{v_{0}}{r_{d}}$ is a nominal yaw angular rate. $p_{r}, p_{d}$, $p_{h}, q_{r}, q_{d}, q_{h}, r_{v}, r_{\omega_{\chi}}$ and $r_{\omega_{\gamma}}$ are constant weighting scalars. $\tilde{d}_{k}$ in Eq. (16) is used to make the angular separation between UAVs to be maintained as $\pi$ radians from the fact that inner product 
of $\mathbf{r}_{k}$ and $\mathbf{r}_{k}^{p}$ is $<\boldsymbol{r}_{k}, \boldsymbol{r}_{k}^{p}>=\boldsymbol{r}_{k}^{T} \boldsymbol{r}_{k}^{p}=\left|\boldsymbol{r}_{k}\right|\left|\boldsymbol{r}_{k}^{p}\right| \cos \triangle \gamma_{k}$ and when $\triangle \gamma_{k}=\pi, \boldsymbol{r}_{k}^{T} \boldsymbol{r}_{k}^{p}+\left|\boldsymbol{r}_{k}\right|\left|\boldsymbol{r}_{k}^{p}\right|=0$, as described in [24]. An adaptive angular separation strategy between relay UAVs (rather than the fixed value $\pi$ ) could be considered to further reduce the number of instances being out of the communication-feasible area; this remains as future work.

The 3-D UAV dynamics described in Eq. (11) can be represented as an equality constraint as:

$$
f_{d}\left(\mathbf{x}_{k}, \mathbf{u}_{k}\right)-\mathbf{x}_{k+1}=0 \text {. }
$$

The collision avoidance requirement between UAVs and obstacles as well as admissible control input ranges can be formulated as the following inequality constraints:

$$
\begin{aligned}
S_{v}\left(\mathbf{u}_{k}\right) & =\frac{\left|u_{v k}-v_{0}\right|-v_{\max }}{v_{\max }} \leq 0 \\
S_{\omega}\left(\mathbf{u}_{k}\right) & =\frac{\left|u_{\omega_{\chi} k}\right|-\omega_{\chi, \max }}{\omega_{\chi, \max }} \leq 0 \\
S_{\omega}\left(\mathbf{u}_{k}\right) & =\frac{\left|u_{\omega_{\gamma} k}\right|-\omega_{\gamma, \max }}{\omega_{\gamma, \max }} \leq 0 \\
S_{c}\left(\mathbf{x}_{k}\right) & =\frac{r_{c}-\left|\boldsymbol{r}_{k}-\boldsymbol{r}_{k}^{p}\right|}{r_{c}} \leq 0 \\
S_{o, l}\left(\mathbf{x}_{k}\right) & =\frac{r_{c}-\left|\boldsymbol{r}_{k}-\boldsymbol{r}_{k}^{o, l}\right|}{r_{c}} \leq 0, \forall l \in\left\{1,2, \ldots, N_{l}\right\}
\end{aligned}
$$

where $\boldsymbol{r}_{k}^{o, l}$ represents the position vector of the $l$-th obstacle (or no-fly zone) among $N_{l}$ obstacles and $r_{c}$ is a safe distance between UAVs or the UAV and obstacles to prevent collision.

\section{NMPC algorithm}

The augmented performance index is defined by incorporating the equality and inequality constraints discussed in the previous section as:

$$
\begin{aligned}
& J_{a}=\phi\left(\tilde{r}_{N}, \tilde{d}_{N}, \tilde{h}_{N}\right)+\sum_{k=0}^{N-1}\left[L\left(\tilde{r}_{k}, \tilde{d}_{k}, \tilde{h}_{k}, \mathbf{u}_{k}\right)+\lambda_{k+1}^{T}\left\{f_{d}\left(\mathbf{x}_{k}, \mathbf{u}_{k}\right)-\mathbf{x}_{k+1}\right\}+\frac{1}{2} \mu_{v} l_{v k} S_{v}^{2}\left(\mathbf{u}_{k}\right)\right. \\
& \left.+\frac{1}{2} \mu_{\omega_{\chi}} l_{\omega_{\chi} k} S_{\omega_{\chi}}^{2}\left(\mathbf{u}_{k}\right)+\frac{1}{2} \mu_{\omega_{\gamma}} l_{\omega_{\gamma} k} S_{\omega_{\gamma}}^{2}\left(\mathbf{u}_{k}\right)+\frac{1}{2} \mu_{c} l_{c k} S_{c}^{2}\left(\mathbf{x}_{k}\right)+\sum_{l=1}^{N_{l}} \frac{1}{2} \mu_{o} l_{o k}\left(S_{o}^{l}\right)^{2}\left(\mathbf{x}_{k}\right)\right]
\end{aligned}
$$

where $\lambda_{k}$ is a Lagrange multiplier, and $\mu_{v}, \mu_{\omega_{\chi}}, \mu_{\omega_{\gamma}}, \mu_{c}$, and $\mu_{o}$ are penalty function parameters. $l_{v k}, l_{\omega_{\chi} k}, l_{\omega_{\gamma} k}, l_{c k}$, and $l_{o k}$ are defined to avoid unnecessary computation for satisfying inequality constraints as:

$$
l_{* k}=\left\{\begin{array}{cc}
0, & S_{*} \leq 0 \\
1, & S_{*}>0
\end{array} .\right.
$$


To derive the optimal control law for the augmented performance index, a Hamiltonian is first defined as:

$$
\begin{aligned}
M_{k} \triangleq & L\left(\tilde{r}_{k}, \tilde{d}_{k}, \tilde{h}_{k}, \mathbf{u}_{k}\right)+\lambda_{k+1}^{T} f_{d}\left(\mathbf{x}_{k}, \mathbf{u}_{k}\right)+\frac{1}{2} \mu_{v} l_{v k} S_{v}^{2}\left(\mathbf{u}_{k}\right)+\frac{1}{2} \mu_{\omega_{\chi}} l_{\omega_{\chi}} S_{\omega_{\chi}}^{2}\left(\mathbf{u}_{k}\right) \\
& +\frac{1}{2} \mu_{\omega_{\gamma}} l_{\omega_{\gamma} k} S_{\omega_{\gamma}}^{2}\left(\mathbf{u}_{k}\right)+\frac{1}{2} \mu_{c} l_{c k} S_{c}^{2}\left(\mathbf{x}_{k}\right)+\sum_{l=1}^{N_{l}} \frac{1}{2} \mu_{o} l_{o k}\left(S_{o}^{l}\right)^{2}\left(\mathbf{x}_{k}\right) .
\end{aligned}
$$

The, the variation of the augmented performance index from Eq. (24) is represented as:

$d J_{a}=\left(\frac{\partial \phi\left(\tilde{r}_{N}, \tilde{d}_{N}, \tilde{h}_{N}\right)}{\partial \mathbf{x}_{N}}-\lambda_{N}^{T}\right) d \mathbf{x}_{N}+\sum_{k=1}^{N-1}\left[\left(\frac{\partial M_{k}}{\partial \mathbf{x}_{k}}-\lambda_{k}^{T}\right) d \mathbf{x}_{k}+\frac{\partial M_{k}}{\partial \mathbf{u}_{k}} d \mathbf{u}_{k}\right]+\frac{\partial M_{0}}{\partial \mathbf{x}_{0}} d \mathbf{x}_{0}+\frac{\partial M_{0}}{\partial \mathbf{u}_{0}} d \mathbf{u}_{0}$.

By selecting the following Lagrange multiplier:

$$
\begin{aligned}
\lambda_{N}^{T} & =\frac{\partial \phi\left(\tilde{r}_{N}, \tilde{d}_{N}, \tilde{h}_{N}\right)}{\partial \mathbf{x}_{N}} \\
\lambda_{k}^{T} & =\frac{\partial M_{k}}{\partial \mathbf{x}_{k}}, \quad \forall k=N-1, \ldots, 0,
\end{aligned}
$$

the variation of $J_{a}$ is simplified to:

$$
d J_{a}=\sum_{k=0}^{N-1} \frac{\partial M_{k}}{\partial \mathbf{u}_{k}} d \mathbf{u}_{k}+\lambda_{0}^{T} d \mathbf{x}_{0}
$$

The right-hand side of Eq. (28) is derived using Eq. (13) as:

$$
\frac{\partial \phi\left(\tilde{r}_{N}, \tilde{d}_{N}, \tilde{h}_{N}\right)}{\partial \mathbf{x}_{N}}=p_{r} \tilde{r}_{N} \frac{\partial \tilde{r}_{N}}{\partial \mathbf{x}_{N}}+p_{d} \tilde{d}_{N} \frac{\partial \tilde{d}_{N}}{\partial \mathbf{x}_{N}}+p_{h} \tilde{h}_{N} \frac{\partial \tilde{h}_{N}}{\partial \mathbf{x}_{N}} .
$$

Besides, the right-hand side of Eq. (29) is derived using Eq. (26) as:

$$
\begin{array}{r}
\frac{\partial M_{k}}{\partial \mathbf{x}_{k}}=q_{r} \tilde{r}_{k} \frac{\partial \tilde{r}_{k}}{\partial \mathbf{x}_{k}}+q_{d} \tilde{d}_{k} \frac{\partial \tilde{d}_{k}}{\partial \mathbf{x}_{k}}+q_{h} \tilde{h}_{k} \frac{\partial \tilde{h}_{k}}{\partial \mathbf{x}_{k}}+\lambda_{k+1}^{T} \frac{\partial f_{d}\left(\mathbf{x}_{k}, \mathbf{u}_{k}\right)}{\partial \mathbf{x}_{k}} \\
+\mu_{c} l_{c k} S_{c}\left(\mathbf{x}_{k}\right) \frac{\partial S_{c}\left(\mathbf{x}_{k}\right)}{\partial \mathbf{x}_{k}}+\sum_{l=1}^{N_{l}} \mu_{o} l_{o k} S_{o}^{l} \frac{\partial S_{o}^{l}\left(\mathbf{x}_{k}\right)}{\partial \mathbf{x}_{k}} .
\end{array}
$$

Jacobians in Eq. (31) can be obtained by the definitions discussed in the previous section. By substituting $d \mathbf{u}_{k}$ into Eq. (30) with the following equation which is basically a gradient descent method to minimise $M_{k}$

$$
d \mathbf{u}_{k}=-\Delta_{k} \frac{\partial M_{k}^{T}}{\partial \mathbf{u}_{k}}
$$

following decreasing variation of $J_{a}$ can be obtained.

$$
d J_{a}=-\sum_{k=0}^{N-1} \Delta_{k} \frac{\partial M_{k}}{\partial \mathbf{u}_{k}} \frac{\partial M_{k}^{T}}{\partial \mathbf{u}_{k}}+\lambda_{0}^{T} d \mathbf{x}_{0} .
$$


Therefore, the control input can be updated using Eq. (33) finally as:

$$
\mathbf{u}_{k}^{i+1}=\mathbf{u}_{k}^{i}-\Delta_{k} \frac{\partial M_{k}^{T}}{\partial \mathbf{u}_{k}}, \quad \forall k=0, \ldots, N-1
$$

where $i$ is the iteration index, and $\Delta_{k}$ is a gradient step size which will determine the convergence speed and solution accuracy. $\frac{\partial M_{k}}{\partial \mathbf{u}_{k}}$ is defined as:

$$
\left(\frac{\partial M_{k}}{\partial \mathbf{u}_{k}}\right)^{T}=\left[\begin{array}{c}
\frac{r_{v}}{v_{\max }^{2}}\left(u_{v k}-v_{0}\right) \\
\frac{r_{\omega_{\chi}}}{\omega_{\chi, \text { max }}^{2}}\left(u_{\omega_{\chi} k}-\frac{v_{0}}{r_{d}}\right) \\
\frac{r_{\omega_{\gamma}}}{\omega_{\gamma, \max }^{2}} u_{\omega_{\gamma} k}
\end{array}\right]+\lambda_{k+1}^{T} \frac{\partial f_{d}\left(\mathbf{x}_{k}, \mathbf{u}_{k}\right)}{\partial \mathbf{u}_{k}}+\left[\begin{array}{c}
\frac{\mu_{v}}{v_{\max }^{2}} l_{v k} S_{v}\left(\mathbf{u}_{k}\right) \operatorname{sign}\left(u_{v k}-v_{0}\right) \\
\frac{\mu_{\omega_{\chi}}}{\omega_{\chi, \max }^{2}} l_{\omega_{\chi} k} S_{\omega_{\chi}}\left(\mathbf{u}_{k}\right) \operatorname{sgn}\left(u_{\omega_{\chi} k}-\frac{v_{0}}{r_{d}}\right) \\
\frac{\mu_{\omega_{\gamma}}}{\omega_{\gamma, \max }^{2}} l_{\omega_{\gamma} k} S_{\omega_{\gamma}}\left(\mathbf{u}_{k}\right) \operatorname{sgn}\left(u_{\omega_{\gamma} k}\right)
\end{array}\right]
$$

where

$$
\frac{\partial f_{d}\left(\mathbf{x}_{k}, \mathbf{u}_{k}\right)}{\partial \mathbf{u}_{k}}=\left[\begin{array}{ccc} 
& \mathbf{0}_{5 \times 3} & \\
\frac{1}{\tau_{v}} T_{s} & 0 & 0 \\
0 & \frac{1}{\tau_{\omega_{\chi}}} T_{s} & 0 \\
0 & 0 & \frac{1}{\tau_{\omega_{\gamma}}} T_{s}
\end{array}\right] .
$$

Note that the iteration continues until a certain stop condition is met (e.g. maximum number of iteration is reached or there is little change compared with the previous step). The detailed online optimization has the following procedures: the initialisation of control input over a moving time horizon, the computation of an augmented performance index, the finding of a Lagrange multiplier by backward integration, the computation of a Jacobian matrix of Hamiltonian $M_{k}$ with respect to the control input, and the update of control inputs. As a practical way, the initial guess of control inputs over the moving time horizon at the first sampling $k=0$ can use a desired nominal speed of the UAV and a desired angular velocity for the standoff orbit at the current height as:

$$
U_{0}^{0}=\left\{\mathbf{u}_{0}, \mathbf{u}_{0}, \ldots, \mathbf{u}_{0}\right\} \in \Re^{3 \times N}
$$

where $\mathbf{u}_{0}=\left(u_{v 0}, u_{\omega_{\chi} 0}, u_{\omega_{\gamma} 0}\right)^{T}, u_{v 0}=v_{0}, u_{\omega_{\chi} 0}=\frac{v_{0}}{r}$ and $u_{\omega_{\gamma} 0}=0$. When $k>0$, the initialisation is done by shifting the optimised control history from the previous sampling $U_{k-1}=\left\{\mathbf{u}_{0}, \mathbf{u}_{1}, \ldots, \mathbf{u}_{N-2}, \mathbf{u}_{N-1}\right\}$ as:

$$
U_{k}^{0}=\left\{\mathbf{u}_{1}, \mathbf{u}_{2}, \ldots, \mathbf{u}_{N-2}, \mathbf{u}_{N-1}, \mathbf{u}_{N-1}\right\}
$$

Although there has been extensive research for stability and convergence of model predictive control [34]-[38], formal analysis for stability of general model predictive control for nonlinear 
systems is far from complete, and the necessary assumptions such as global boundness of a cost function do not hold in our nonconvex and constrained nonlinear optimisation problem. In particular, since our problem involves the time-varying reference as the standoff orbit unpredictably moves with a moving ground convoy and surrounding buildings, it is difficult to determine terminal constraints [38] or a control Lyapunov function [36] needed to guarantee a certain degree of stability. Thus, instead of the formal methods guaranteeing the convergence, this study uses several numerical simulations to validate the performance and convergence property of the proposed approach in the next section. Note that deliberation of the optimality over the moving time horizon $N$ renders our predictive-based approach less prone to the local minima than greedy potential function-based methods. To this end, we would need $N$ to be large enough to predict over the local minima at the expense of the computation time [39].

Note that there are many other advanced optimisation algorithms and high-performance solvers to tackle the nonlinear and nonconvex optimisation problem defined in Eq. (12) such as IPOPT and SNOPT [40], [41]. As these solvers are fairly lightweight and optimised in terms of performance (e.g., computation time and optimality), we could directly use them. However, in this paper, we decided to use a basic gradient-based algorithm derived from the optimal control law rather than the generalised solver in order not to be dependent on the third-party software. The current work is for a feasibility study, and performance optimisation remains as future work when applying the proposed approach to a real system.

\section{Numerical Simulations}

This section performs numerical simulations using the proposed NMPC-based convoy following trajectory planning for a moving ground convoy. The simulation sampling time $T_{s}$ is set to $0.5 \mathrm{~s}$ and the parameter setting for simulations is shown in Table I. Three sample convoy following scenarios are considered where the convoy moves through the urban area surrounded by a number of buildings as shown in Fig. 7. A real urban map of Zurich in Switzerland is used. As mentioned in Section II, the map environment and convoy paths are assumed to be known.

Figure 8 displays NMPC-based communication-aware convoy following trajectory planning results for the Case 1 scenario with an additionally introduced no-fly zone. It includes UAV trajectories from different views, loitering orbit distance tracking error, height tracking error, phase angle (angular separation) difference between UAVs, and time histories if the following 
TABLE I

SIMULATION PARAMETERS

\begin{tabular}{llc} 
Parameter & Value & Unit \\
\hline$\gamma_{d}$ & $\pi$ & $\mathrm{rad}$ \\
$\left(v_{0}, v_{\max }\right)$ & $(20,10)$ & $\mathrm{m} / \mathrm{s}$ \\
$\left(r_{d}, r_{c}\right)$ & $(45,50)$ & $\mathrm{m}$ \\
$\left(\omega_{\phi, \max }, \omega_{\gamma, \max }\right)$ & $(0.6,0.15)$ & $\mathrm{rad} / \mathrm{s}$ \\
$\tau_{v}, \tau_{\omega_{\phi}}, \tau_{\omega_{\gamma}}$ & $1 / 3$ & $\mathrm{sec}$ \\
$N$ & $6($ equivalent to $3 \mathrm{secs})$ & N/A \\
$\left(p_{r}, p_{d}, p_{h}, q_{r}, q_{d}, q_{h}\right)$ & $\left(2 e 2,4 e 2,2 e 3, p_{r} / N, p_{d} / N, p_{h} / N\right)$ & N/A \\
$\left(r_{v}, r_{\omega_{\phi}}, r_{\omega_{\gamma}}\right)$ & $(1 e 2,5 e 1,1 e 1)$ & N/A \\
$\left(\mu_{v}, \mu_{\omega_{\phi}}, \mu_{\omega_{\gamma}}, \mu_{c}\right)$ & $(1,1 e 3,1 e 3,1 e 4)$ & N/A
\end{tabular}

UAV is in or out of the communication-feasible area. Note that, in addition to two convoy following relay UAVs (blue and red lines), the intermediate relay UAV is included which loiters around the medium point of the line connecting the GCS and the convoy considering the maximum communication range (300 $\mathrm{m}$ in this study). As mentioned in Section II, detailed trajectory design of the intermediate relay UAV is not considered in this study. Figure 9 shows the sample capture of a convoy following simulation for the Case 1 scenario with different views at a certain time instant to facilitate the reader's understanding. As shown in Fig. 9(c) and (d), two following UAVs are flying within the cross section of the communication feasible area by accurately following the desired loitering orbit while avoiding the no-fly zone by looking ahead multiple steps.

From Figs. 8 and 9, it can be easily observed that the centre of the desired loitering orbit does not coincide with the ground convoy position horizontally as shown in Fig. 9(a) and (d) due to the fact that the LOS obstruction by surrounding buildings skews the communication-feasible area. This means that the mission of UAVs is not just simply following the convoy directly above it; it is rather complicated as UAVs need to stay within the communication-feasible area to avoid the LOS block while following the moving convoy. Besides, since relay UAVs accurately track the desired loitering orbit and the height while maintaining their desired phase angle (180 deg), UAVs are within the communication-feasible area during most of the simulation time (except at around $20 \mathrm{~s}$ to avoid the no-fly zone), as shown in Fig. 8(f). Although there are a few time 
instances where one of UAVs is outside the communication-feasible area, none of them lose the communication for both UAVs at the same time in this scenario.

To verify the feasibility and benefits of the proposed approach, three case scenarios (without no-fly zones) explained above were compared against the broadly-used LVFG (Lyapunov Vector Field Guidance) [26], [30]. Here, the LVFG uses the vector field function to compute the desired heading angle for the UAV to follow, which makes the UAV loiter around the centre of the desired loitering orbit. The LVFG uses a decoupled one-step ahead feedback control structure: i) the heading control for loitering orbit distance keeping which guides the UAV onto the generated stable orbit around the centre of the desired loitering orbit, ii) the speed control for phase angle keeping on the same orbit, and iii) the flight path angle control to follow the desired orbit height. Details for this LVFG can be found in [26], [27]. Meanwhile, the NMPC guidance (trajectory planning) utilises the coupled sub-optimal control commands for heading, speed and flight path angle using Eq. (12) computed over the receding horizon (multiple time steps) ahead with the known future convoy trajectory and the corresponding communication-feasible area.

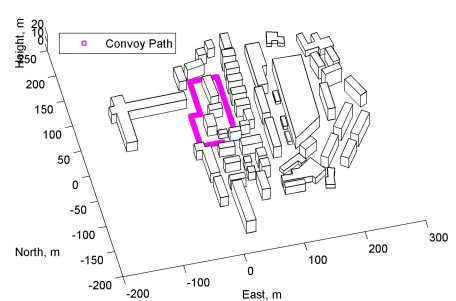

(a) Case 1 (70 seconds)

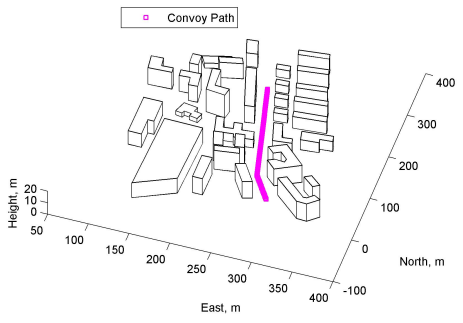

(b) Case 2 (45 seconds)

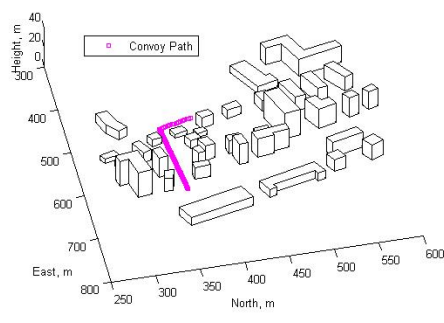

(c) Case 3 (40 seconds)

Fig. 7. Sample convoy following scenarios where $(\cdot)$ represents the duration of a convoy mission.

Table II compares tracking guidance performance in terms of the distance from the desired loitering orbit (i.e. $\left|r-r_{d}\right|$ ), height, phase keeping, control efforts, and the number of instances for the UAV being out of the communication-feasible area. The control efforts (consumptions) of $u_{v}, u_{\chi}$, and $u_{\gamma}$ are computed by integrating the time histories of $\left|u_{v}-v_{o}\right|,\left|u_{\chi}\right|$ and $\left|u_{\gamma}\right|$, respectively. Firstly, by using two UAVs, the number of 'out of communication' instances are significantly reduced compared with using a single UAV for both the LVFG and the NMPC trajectory planning. Besides, the proposed NMPC shows much better performance in terms of 


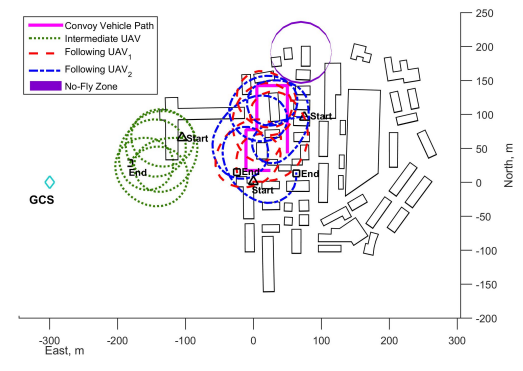

(a) Top-down view with UAV trajectories

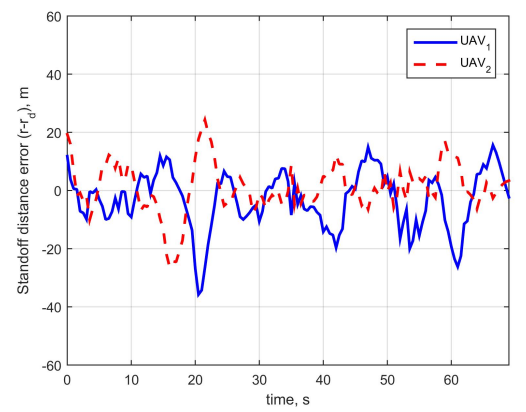

(c) Loitering orbit distance error evolution

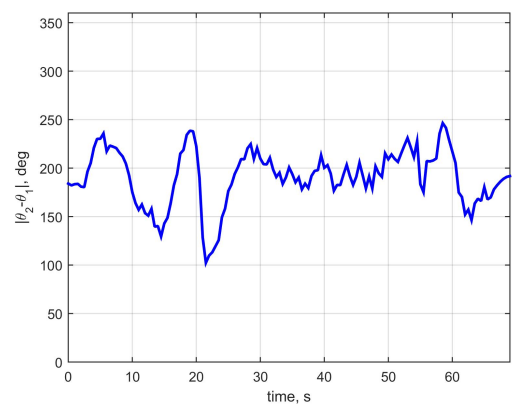

(e) Phase angle difference of UAVs

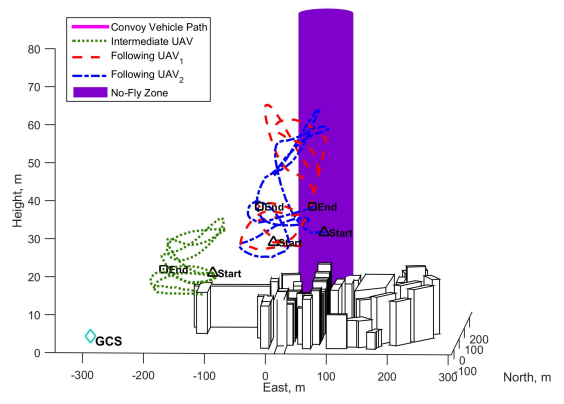

(b) 3-D view with UAV trajectories

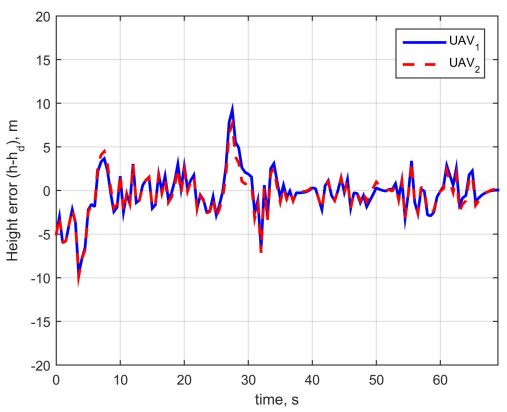

(d) Height error

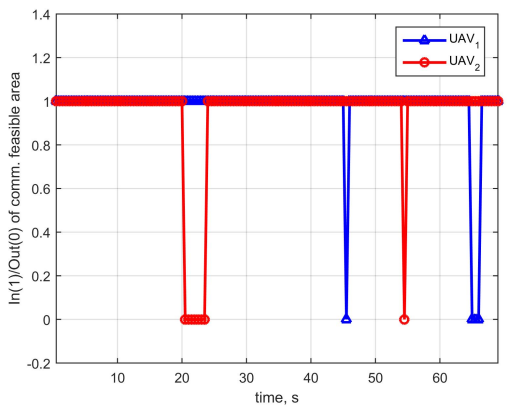

(f) In/out of communication-feasible area

Fig. 8. NMPC-based communication-aware convoy following trajectory planning simulation results for the Case 1 scenario.

tracking the loitering orbit (i.e. orbit distance) and height and consequently the relay task (i.e. fewer 'out of communication' instances). The NMPC approach also use much less control efforts. This benefit comes from computing coupled optimal control commands (among speed, heading rate, and pitch rate) with future (predicted) information on the desired loitering orbit and UAV kinematic constraints in the optimisation process. 


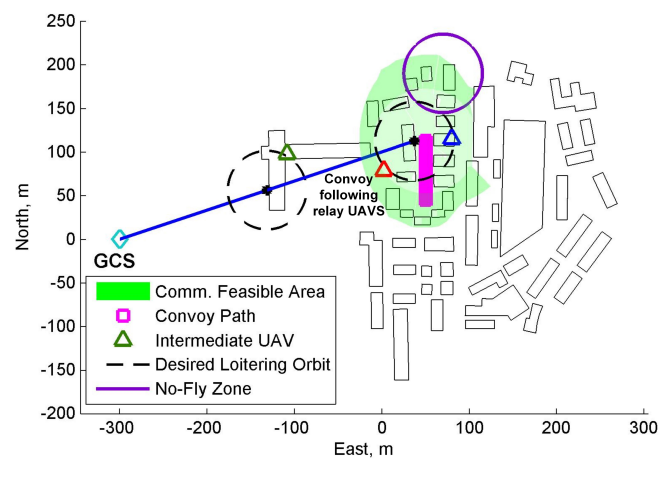

(a) Top view

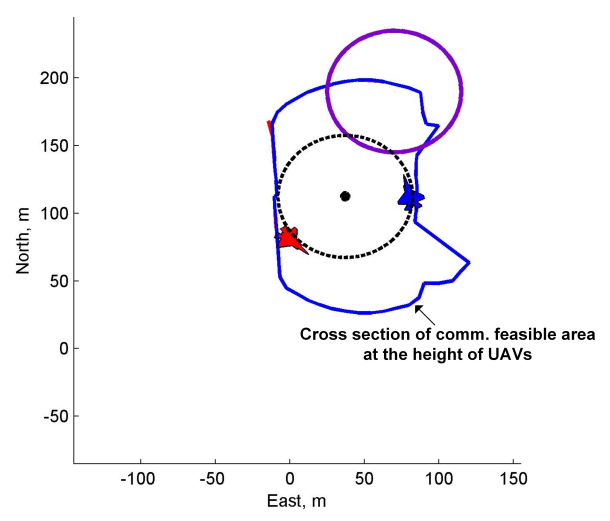

(c) Cross section view

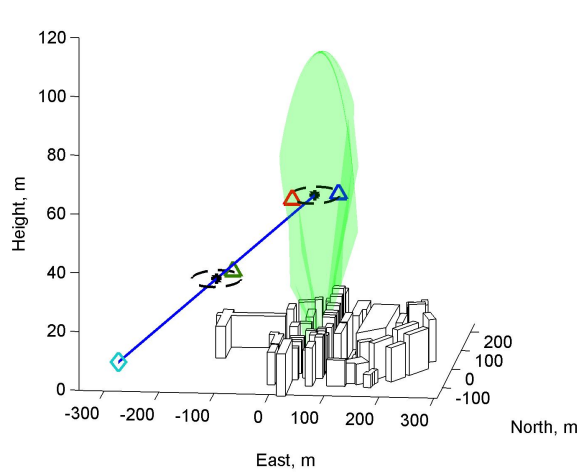

(b) 3-D view

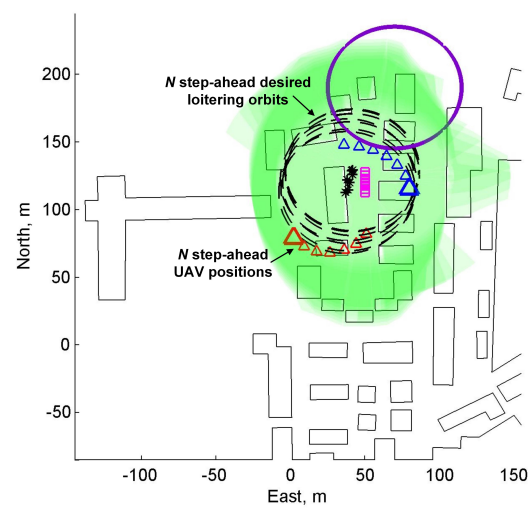

(d) Receding horizon view

Fig. 9. Sample capture of convoy following (Case 1) with different views. Red and blue triangle represent the convoy following/relay UAVs, and asterisk symbols in (d) represent the centre of the desired loitering orbits for the multiple time steps.

The mean computation time of each UAV's NMPC routine at every sampling is about $0.3 \mathrm{~s}$ when using an Intel Core i7-6700 CPU with $3.40 \mathrm{GHz}$ with 16 GB RAM in a Matlab code. Although this is much slower than that (less than $1 \mathrm{~ms}$ ) of the LVFG, it could be considered reasonable since the NMPC routine provides guidance commands. In general, the update rate of guidance commands could be much slower (e.g. $0.5 \mathrm{~s}$ set in this study) than that of the lowlevel flight control commands. Besides, the computation time could be further reduced by tuning optimisation parameters at the expense of tracking performance or if written in a $\mathrm{C} / \mathrm{C}++$ code.

As a final remark, considering future positions of the ground convoy is really useful for planning the trajectory in complex urban environments. Since the proposed NMPC-based ap- 
TABLE II

TRACKING PERFORMANCE FOR SAMPLE SCENARIOS

\begin{tabular}{lcccccc}
\hline \multirow{2}{*}{ Mean error } & \multicolumn{2}{c}{ Case 1} & \multicolumn{2}{c}{ Case 2 } & \multicolumn{2}{c}{ Case 3 } \\
& LVFG & NMPC & LVFG & NMPC & LVFG & NMPC \\
\hline \hline Orbit distance (m) & 10.51 & 5.11 & 10.72 & 6.76 & 13.10 & 6.35 \\
Height $(\mathrm{m})$ & 5.80 & 1.64 & 6.77 & 3.44 & 5.41 & 1.56 \\
Angular separation $(\mathrm{deg})$ & 23.05 & 27.95 & 31.36 & 28.82 & 24.22 & 20.13 \\
\hline$u_{v}$ control efforts $(\mathrm{m} / \mathrm{s})$ & 111.53 & 75.47 & 95.75 & 75.29 & 67.87 & 27.99 \\
$u_{\chi}$ control efforts $(\mathrm{rad} / \mathrm{s})$ & 28.69 & 28.49 & 17.57 & 16.93 & 16.45 & 15.99 \\
$u_{\gamma}$ control efforts $(\mathrm{rad} / \mathrm{s})$ & 10.19 & 3.73 & 6.60 & 4.18 & 5.80 & 2.09 \\
\hline Out of Communication* & $28 / 27(5)$ & $1 / 1(0)$ & $26 / 14(9)$ & $2 / 3(0)$ & $14 / 16(1)$ & $2 / 2(0)$ \\
\hline
\end{tabular}

*UAV1/UAV2 (out of comm. for both UAVs at the same time)

proach utilises the predicted future position of the ground convoy, we can obtain a good tracking performance where the benefit can be maximised with the cooperating ground convoy. If there is no unexpected situation or pop-up threat on the ground or in the air, the proposed approach will provide a better result than that of the one-step look ahead algorithm (e.g. LVFG or similar methods) as long as the receding horizon window (i.e. look-ahead step size $N$ ) is big enough. Note that, however, a too big horizon window considering the distant future makes the UAV fly too proactively resulting in an unsatisfactory tracking performance; thus, the size of window needs to be carefully determined depending on the UAV's dynamic capability and the urban environment. A short video including some numerical simulation results is provided at the following link: https://www.dropbox.com/s/c7r5pzp4t13t82x/Comm_Relay_Movie.zip?dl=0. For the flight test, due to the indoor space limitation, we used a quadrotor UAV which attempts to stay at the centre the desired loitering orbit (rather than loitering around it). Following the centre of the desired orbit is still meaningful to make the trajectory planning algorithm robust to the navigational position error or position control error since as long as a quadrotor is within the loitering orbit, communication to the convoy is expected to be guaranteed.

\section{CONCLUSIONS}

This paper has proposed a communication-aware trajectory planning approach which makes relay UAVs stay within the communication-feasible area against a moving ground convoy in a 
complex urban environment to ensure communication between the ground control station and the convoy. Numerical simulations showed the feasibility and benefit of the proposed approach based on the nonlinear model predictive control method which utilises future information and provides coupled sub-optimal control commands. The approach proposed in this study can be beneficial for cooperative/coordinated operations of unmanned aerial and ground vehicles in an urban disaster area, especially when humans cannot directly enter it due to a possible hazard such as radioactivity or contamination. This work can also be applied to a visual target tracking problem in order to persistently track the moving target while maintaining a visual line-of-sight.

An adaptive angular or spatial separation between relay UAVs (rather than the fixed value $\pi$ with the same standoff distance used in this study) will be investigated to further reduce the number of out of communication instances. A more realistic communication model which considers not only the LOS condition but also other communication properties such as multipath effects due to diffraction and reflection in the complex urban environment will be studied. Robust trajectory planning under uncertainty on a convoy path or an urban map will also be followed as future work.

\section{ACKNOWLEDGMENTS}

This work was supported by Basic Science Research Program through the Lockheed Martin Corporation Republic of Korea Science, Technology, Research (RoKST\&R) Initiative, the 2018 Research Fund (1.180015.01) of UNIST (Ulsan National Institute of Science and Technology), and the UK Engineering and Physical Science Research Council (EPSRC) under the Grant $\mathrm{EP} / \mathrm{J} 011525 / 1$.

\section{REFERENCES}

[1] T. Samad, J.S. Bay, and D. Godbole. Network-centric systems for military operations in urban terrain: The role of UAVs. Proceedings of the IEEE, 95(1):92-107, 2007. DOI: 10.1109/JPROC.2006.887327.

[2] H.S. Shin and P. Segui-Gasco. UAV Swarms: Decision-Making Paradigms. Encyclopedia of Aerospace Engineering. Wiley \& Sons, Ltd., 2014.

[3] P. Basu, J. Redi, and V. Shurbanov. Coordinated flocking of UAVs for improved connectivity of mobile ground nodes. In IEEE Military Communications Conference (MILCOM 2004), 2004.

[4] S. Kim, P. Silson, A. Tsourdos, and M. Shanmugavel. Dubins path planning of multiple unmanned airborne vehicles for communication relay. Proceedings of the Institution of Mechanical Engineers, Part G: Journal of Aerospace Engineering, 225(1):12-25, 2011. DOI: 10.1243/2041302510JAERO848. 
[5] S.S. Ponda, L.B. Johnson, A.N. Kopeikin, H.L. Choi, and J.P. How. Distributed planning strategies to ensure network connectivity for dynamic heterogeneous teams. IEEE Journal on Selected Areas in Communications, 30(5):861-869, 2012. DOI: $10.1109 /$ JSAC.2012.120603.

[6] J.J. Acecedo, C. Begona, I. Maza, and A. Ollero. Distributed approach for coverage and patrolling missions with a team of heterogeneous aerial robots under communication constraints. International Journal of Advanced Robotic Systems, 10(28):1-13, 2013.

[7] K.J. Obermeyer, A. Ganguli, and F. Bullo. Multi-agent deployment for visibility coverage in polygonal environments with holes. International Journal on Robust and Nonlinear Control, 21(12):1467-1492, 2011. DOI: 10.1002/rnc.1700.

[8] H. Zhu, A.L. Swindlehurst, and K. Liu. Optimization of MANET connectivity via smart deployment/movement of unmanned air vehicles. IEEE Transactions on Vehicular Technology, 58(7):3533-3546, 2009.

[9] S. Kim, H. Oh, J. Suk, and A. Tsourdos. Coordinated trajectory planning for efficient communication rely using multiple UAVs. Control Engineering Practice, 29:42-49, 2014.

[10] S. Hauert, L. Winkler, J.C. Zufferey, and D. Floreano. Ant-based swarming with positionless micro air vehicles for communication relay. Swarm Intelligence, 2:167-188, 2008. DOI: 10.1007/s11721-008-0013-5.

[11] D.H. Choi, S.H. Kim, and D.K. Sung. Energy-efficient maneuvering and communication of a single UAV-based relay. IEEE Transactions on Aerospace and Electronic Systems, 50(3):2320 - 2327, 2014.

[12] M. Stachura and E. Frew. Communication-aware information-gathering experiments with an unmanned aircraft system. Journal of Field Robotics, pages 1-21, 2017. DOI: 10.1002/rob.21666.

[13] A. Al-Hourani, S. Kandeepan, and S. Lardner. Optimal lap altitude for maximum coverage. IEEE Wireless Communications Letters, 3:569-572, 2014. 6.

[14] A. Al-Hourani, S. Kandeepan, and A. Jamalipour. Modeling air-to-ground path loss for low altitude platforms in urban environments. In IEEE Global Communications Conference (GLOBECOM), Austin, TX, USA, Feb 2014.

[15] J. Chen, U. Yatnalli, and D. Gesbert. Learning radio maps for UAV-aided wireless networks: A segmented regression approach. In IEEE International Conference on Communications, Paris, France, May 2017.

[16] J. Chen and D. Gesbert. Optimal positioning of flying relays for wireless networks: A LOS map approach. In IEEE International Conference on Communications, Paris, France, May 2017.

[17] E.W. Frew and T.X. Brown. Airborne communication networks for small unmanned aircraft systems. Proceedings of the IEEE, 96(12):2008-2027, 2008. DOI: 10.1109/JPROC.2008.2006127.

[18] J. Le Ny, A. Ribeiro, and G.J. Papas. Adaptive communication-constrained deployment of unmanned vehicle systems. IEEE Journal on Selected Areas in Communications, 30(5):923-934, 2012.

[19] D.H. Choi, B.H. Jung, and D.K. Sung. Low-complexity maneuvering control of a UAV-based relay without location information of mobile ground nodes. In IEEE Symposium on Computers and Communication (ISCC), Funchal, Portugal, 2014.

[20] P. Ladosz, H. Oh, and W. H. Chen. Prediction of air-to-ground communication strength for relay uav trajectory planner in urban environments. In IEEE International Conference on Intelligent Robots and Systems, Vancouver, Canada, Sep 2017.

[21] S. Watza, R. K. Rajasekaran, and E. Frew. Hybrid rf propagation model using itm and gaussian processes for communication-aware planning. In Robotics: Science and Systems (RSS), Massachusetts, USA, July 2017.

[22] Y. Gao, Y. Li, C. Lyu, and Y. Liu. Autonomous wi-fi relay placement with mobile robots. IEEE Transactions on Mechatronics, 22:2532-2542, 2017. 6. 
[23] J. Kim and Y. Kim. Moving ground target tracking on dense obstacle area using UAVs. In 17th IFAC World Congress, Seoul, Korea, 2008.

[24] S. Kim, H. Oh, and A. Tsourdos. Nonlinear model predictive coordinated standoff tracking of moving ground vehicle. Journal of Guidance, Control and Dynamics, 36(2):557-566, 2013. DOI: 10.2514/1.56254.

[25] D.B.E. Medagoda and P.W. Gibbens. Multiple horizon model predictive flight control. Journal of Guidance, Control, and Dynamics, 37(3):946-951, 2014. DOI: 10.2514/1.62889.

[26] E.W. Frew, D.A. Lawrence, and S. Morris. Coordinated standoff tracking of moving targets using Lyapunov guidance vector fields. Journal of Guidance, Control, and Dynamics, 31(2), 2008. DOI: 10.2514/1.30507.

[27] H. Oh, S. Kim, H. Shin, P. Ladosz, and A. Tsourdos. Communication-aware convoy following guidance for UAVs in a complex urban environment. In The 24th Mediterranean Conference on Control and Automation, 2016. DOI: 10.1109/MED.2016.7535884.

[28] V. Shaferman and T. Shima. Unmanned aerial vehicles cooperative tracking of moving ground target in urban environments. Journal of Guidance, Control and Dynamics, 31(5), 2008. DOI: 10.2514/1.33721.

[29] K. J. Obermeyer and Contributors. The VisiLibity library. http://www.VisiLibity.org, 2008 . R-1.

[30] H. Oh, S. Kim, H. Shin, and A. Tsourdos. Coordinated standoff tracking of moving target groups using multiple UAVs. IEEE Transactions on Aerospace and Electronic Systems, 51(2):1501-1514, 2015. DOI: 10.1109/TAES.2015.140044.

[31] H. Chen. UAV path planning with tangent-plus-lyapunov vector field guidance and obstacle avoidance. IEEE Transactions on Aerospace and Electronic Systems, 49(2):840-856, 2013. DOI: 10.1109/TAES.2013.6494384.

[32] S. Park. Guidance law for standoff tracking of a moving object. Journal of Guidance, Control and Dynamics, 2017. DOI: 10.2514/1.G002707.

[33] C.E. Rasmussen and C.K.I. Williams. Gaussian Process for Machine Learning. MIT Press, 2006. Chapter 2.

[34] W.H. Chen, D.J Ballance, and J. O'Reilly. Model predictive control of nonlinear systems: computational burden and stability. IEE Control Theory and Applications, 147(4):387-394, 2000.

[35] J. Shin and H. Kim. Nonlinear model predictive formation flight. IEEE Transactions on Systems, Man, and Cybernetics-Part A: Systems an Humans, 39(5), 2009.

[36] A. Jadbabaie, J. Yu, and J. Hauser. Unconstrained receding-horizon control of nonlinear systems. IEEE Transactions on Automatic Control, 46:546-558, 2001. 5.

[37] S. S. Keerthi and E. G. Gilbert. Optimal infinite-horizon feedback laws for a general class of constrained discrete-time systems: Stability and moving-horizon approximations. Journal of Optimization Theory and Applications, 57:265-293, 1988. 2 .

[38] H. Michalska and D.Q. Mayne. Robust receding horizon control of constrained nonlinear systems. IEEE Transactions on Automatic Control, 38:1623-1633, 1993. 11.

[39] D.H. Shim, H.J. Kim, and S. Sastry. Decentralised nonlinear model predictive control of multiple flying robots. In IEEE Conference on Decision and Control, Hawaii, USA, Dec 2003.

[40] A. Wachter and L. T. Biegler. On the implementation of a primal-dual interior point filter line search algorithm for large-scale nonlinear programming. Mathematical Programming, 106(1):25-57, 2006.

[41] P. E. Gill, W. Murray, , and M. A. Saunders. SNOPT: An SQP algorithm for large-scale constrained optimization. SIAM Review, 47(1):99-131, 2005. 